\title{
The social nature of disability, disease and genetics: a response to Gillam, Persson, Holtug, Draper and Chadwick
}

\author{
Christopher Newell School of Medicine, University of Tasmania
}

\begin{abstract}
The dominance of the biomedically informed view of disability, genetics, and diagnosis is explored. An understanding of the social nature of disability and genetics, especially in terms of oppression, adds a richer dimension to an understanding of ethical issues pertaining to genetics. This is much wider than the limited question of whether or not such technology discriminates. Instead, it is proposed that such technology will perpetuate the oppression and control of people with disability, especially if the knowledge of people with disability is not utilised in bioethical debates.
\end{abstract}

(Fournal of Medical Ethics 1999;25:172-175)

Keywords: Disability; deaf culture; social; reproductive technology

\section{The important social aspects}

This response to Gillam, Holtug, Persson, and Draper and Chadwick, seeks to show the dominance of the biomedically informed view of disability, genetics, and diagnosis. According to such a view developments in genetic technology are inherently good. Even those who argue that technology is neutral would see its application to eliminate the suffering of individuals as inherently good. Yet, this world view has been predominantly constructed by those who do not live a life of disability. Most bioethical discourse is informed by the medical model. Despite the rise of postmodernism, bioethics has yet adequately to recognise the social nature of disability, genetics, ethics, and scientific knowledge. Hence, I seek to show the social dimension of genetics, referred to by Gillam and made more explicit by Draper and Chadwick. Such knowledge is explicitly to be found in the critical disability studies literature.

\section{Discrimination or oppression?}

Lynn Gillam asks the question as to whether prenatal diagnosis constitutes discrimination against "the disabled". Gillam makes a strong case that prenatal diagnosis does not inherently discrimi- nate against people with disability. This would much stronger if it was allowed that prenatal dia nosis may apply to all conditions such as sex, skon colour or any other attribute. Provided such techin nology is utilised for all sorts of purposes rathest than just screening disability, she makes a good case.

However, the choice of question is indicative $\frac{F}{\partial}$ the political dimension of ethical debates. F\&: example, Gillam's paper is remarkably similar one presented at a conference in 1994. At the time I suggested that the important question was not to do with discrimination, but oppression. ${ }^{1}$ that paper I argued the social nature of disabilit proposing that prenatal diagnosis and termination is a technology of oppression and control which serves to devalue the lives of people identified having disabilities. Certainly the critical disabili studies literature ${ }^{2}$ upholds oppression as a usef concept.

Much of the bioethical and scientific literature accepts disability as a given. Most medical tex $\mathbb{B}_{3}$ use disease labels to identify disability states and quality of life. For example, in Australia thie National Health and Medical Research Council 兽 a 1988 discussion paper, The Ethics of Limiting Life-Sustaining Treatment, argued for "nok intervention" of "severely affected children" such as those with severe spina bifida. Yet, suct views tend to be written without utilising the knowledge of those who live in the socio-politica space of disability. ${ }^{4}$

A relatively rare perspective is provided by AI son Davis. She identifies as having severe spir bifida, and writes:

“... if I lived in a society where being in wheelchair was no more remarkable than wearin glasses and if the community was completer accepting and accessible, my disability would b8 an inconvenience and not much more than that. is society which handicaps me, far more serioushy and completely than the fact that I have sping bifida." 
As Davis also writes, in reviewing Kuhse and Singer's book, Should the Baby Live?:

"I was born with severe spina bifida, and am confined to a wheelchair as a result. Despite my disability and the gloomy predictions made by doctors at my birth, I am now leading a very full, happy and satisfying life by any standards. I am most definitely glad to be alive. Yet, because handicapped people are now presumed by some doctors, philosophers and Society in general to have the capacity only for being miserable and an economic burden on the community most of those who would otherwise grow up to be like me are now aborted or "allowed to die" (such a comfortable euphemism) at birth."

\section{The social nature of disability}

The stories of people like Davis point to the social nature of disability. This is further confirmed by an analysis in terms of disability discourses in the critical disability studies literature. ${ }^{7}$ Rather than people with disability inherently leading lowquality lives, and being an economic burden on society, we need to look at the way such disability is constructed. Those of us with disabilities tend to have to rely upon Social Security because of the way society is ordered, ${ }^{8}$ rather than there being an economic situation which enables and facilitates our contribution to society.

\section{The problem of rejected knowledge}

In Persson, Holtug and Gillam's papers we also encounter the problem of rejected knowledge, mirroring the broader bioethical debate. ${ }^{9}$ Certainly, there is literature which argues contrary to these writers. The questions and analysis proposed by the disability studies literature is markedly different. What of a consideration of such arguments in bioethical debate?

Likewise, few writers in bioethics define "disability" or "abnormality". Yet, this is crucial to our endeavour. I suggest a social constructivist account, but this does not deny a physiological component. I have spent years of my life in hospitals and the symptoms were not just social! Rather, it is the social meanings given to "difference", "the disabled body" and the "disabled mind" which are important in terms of "social construction".

It can also be argued that Holtug, Persson and Gillam discuss those with disability as the objectified other, and tend to ignore the social dimension. For example, Persson holds that disabilities such as Down's syndrome lessen ability to contribute to others.
Yet, one of my friends, John, is identified as having Down's syndrome. Pitied by some, he has a happy existence due in part to the care of others, but which of us does not need care? Some pity him his intelligence (certainly a social construct). However, I sometimes wonder whether he is not more fortunate than some of my academic colleagues as I see them leading stressed and unhealthy lives in artificial environments. Do we sometimes overlook simple truth and beauty in our obsession to control nature, with the attendant ideology of progress and the search for the technological fix? The pleasure John takes in life, and his emphasis on the importance of relationship with others, is far more valuable to me than if he was producing large quantities of capital or academic papers. Likewise I have learnt more about life and the human condition as someone in receipt of care than via my doctoral research.

\section{Whose voice, whose knowledge?}

However, what of Holtug's passionate advocacy for younger people, whose story he tells while proposing genetic enhancements. His moral trump is probably correct: "The chances are that you - the reader of this journal-are a reasonably healthy person". Yet, Holtug tells his patients' stories from his own biomedically informed perspective, rather than the narratives of people with disabilities being told in their own terms. ${ }^{10}$

Like Gillam and Persson, Holtug fails adequately to recognise the social nature of disease. Genetic conditions occur in a social context, and their meaning and impact are inherently social. For example, intelligence varies with cultural setting, as does attraction. The vexing questions are which attributes should be enhanced, and which are to be regarded as defective, inferior or indeed, superior?

Here we must recognise the political nature of our endeavour. It all relates to the age-old questions of "what sort of people should there be" and "who will decide"?" We need an analysis in terms of power relations and a recognition of the exclusion of the voices of people with disability from the debate.

There may well be an argument for genetic enhancements, but our analysis needs an understanding of the social nature of genetics, disability, disease and technology. A case for enhancements flows from the lived experience of severe pain or suffering of people with genetic conditions. Much suffering may be for social reasons. However, for suffering which exists regardless of social setting there may be a case for stem line enhancement. This would probably eliminate claims based upon height or "trivial" claims. 


\section{Old and new problems}

Draper and Chadwick's discussion of preimplantation genetic diagnosis helps us to look at the social dimensions of disability, disease and genetics. For example, the case of Linda and Paul, both deaf, who select congenital deafness in an embryo for implantation.

Many deaf people (predominantly those who are prelingually deaf, use sign language as their first language, and belong to deaf culture) ${ }^{12}$ do not identify as having disability. They hold that society's norms oppress them, identifying as a socio-linguistic minority. In short, there is a conflict between the dominant, hearing, and the deaf world, views. Each world view utilises different premises, and therefore the ethical arguments reach markedly different conclusions.

Draper and Chadwick's article points to the next important step in bioethics. This is to utilise the unfiltered perspectives of people who are the subject of the bioethical gaze, and the subject of care, and to regard such perspectives as important knowledge. To do this we will need to recognise the way in which knowledge is constructed. As Mary Johnson, an American disability activist, notes regarding decision making based on dominant information:

"A decision to abort based on the fact that the child is going to have specific individual characteristics such as mental retardation, or in the case of cystic fibrosis, a build-up of mucus in the lungs, says that those characteristics take precedence over living itself. That they are so important and so negative, that they overpower any positive qualities there might be in being alive." 13

Johnson argues for a society where conditions such as cystic fibrosis are seen as needing to be treated, not feared. There is little tolerance of anything that deviates from narrow norms. Parents and potential parents are faced with stereotypical information. Johnson persuasively argues:

"A disabled fetus represents for parents a problem that may have far more to do with society than with disability. Disabled children confront a hostile environment." 13

There are obvious lessons here for all aspects of the debate to do with genetics, especially in terms of "desirable" and "undesirable" attributes.

All of this, of course, is hardly merely a theoretical issue. What do I say, for example, regarding a decision to abort based upon such a condition as Down's syndrome, like my friend John has? I remember well my wife and I presenting for prenatal care when having a child, and being subject to significant pressure for routine screening. That pressure built when we said wert were not interested in tests which led to. information which could only be used for the put? poses of abortion, as opposed to therapy. John situation helps me to question the dominance of perspective which believes that a likelihood of dis ability is so significant that it acts as a trump care regarding abortion. There are even, for exampl some jurisdictions which allow partial-birth aboti tion on the grounds of disability but no other factor.

It needs also to be recognised that genetie information regarding conditions with a high ris of imminent death or severe pain for a child, bs virtue of the condition, may well have merit i assisting people with difficult choices about reproduction, well prior to the vexed issue of abortion. It is probably, however, the case of tho deaf couple cited by Draper and Chadwick which is most confronting. They choose congenital deaf ness in an embryo for implantation. What would do? I cannot adequately answer as I do not belon to the deaf culture and bring a hearing world view to bear. The task for bioethics is to assist such couple to explore the reasons they choose a course of action. However, it is possible to argue that the dilemma is indicative of the oppression found this technology. If we are not careful then the resolution of this and other dilemmas simp becomes a product of dominant power relations. is only in the real world that we can adequate explore this and other considerations. In the same way that I have called for the use of the knowledge of people with disability, the full involvement the deaf community is crucial to our understand ing and decision making.

\section{The social nature of disability and genetics}

Thus, in this inadequate response, I highlight the social and political dimension of bioethics and genetics, and especially the question of whose knowledge do we count and whose do we reject? An understanding of the social nature of disabilite and genetics, especially in terms of oppression, will add a richer dimension to an understanding of ethical issues pertaining to genetics. This is muct wider than the limited question of whether or not such technology discriminates. Instead, it is proposed that such technology will perpetuate the oppression and control of people with disabilite especially if the knowledge of people with disabie ity is not utilised in bioethical debates.

Christopher Newell, PhD, is Senior Lecturer in the School of Medicine, University of Tasmania, Hobake, Tasmania, Australia. 


\section{References}

1 Newell C. A critique of the construction of prenatal diagnosis and disability. In: McKie J ed. Ethical issues in prenatal diagnosis and the termination of pregnancy. Melbourne: Centre for Human Bioethics, Monash University, 1994: 89-96.

2 Abberley P. The concept of oppression and the development of a social theory of disability. Disability, Handicap and Society 1987;2:5-20.

3 National Health and Medical Research Council. Discussion paper. The ethics of limiting life-sustaining treatment. Canberra: National Health and Medical Research Council, 1988: 5.

4 Newell C. A critical evaluation of the NH\&MRC's The ethics of limiting life-sustaining treatment and related perspectives on the bioethics of disability. Australian Disability Review 1991;4:4657.

5 Davis A. From where I sit. London: Triangle, 1989: 19.

6 Davis A. Yes, the baby should live. New Scientist 1985;31:54.

7 See for example Fulcher G. Disabling policies? London: Falmer Press, 1989.

8 Bowe F. Rehabilitating America. New York: Harper and Row, 1980.

9 For a review of some literature in the history and philosophy of science regarding "rejected knowledge", see for example: Richards E. Vitamin C and cancer. London: MacMillan, 1991; New- ell C. The social construction of the wheelchair and cochlear implant: a study of the definition and regulation of disability. Unpublished PhD thesis. Victoria: Faculty of Arts, Deakin University, 1994; Wallis R, ed. On the margins of science: the social construction of rejected knowledge. Keele, Staffordshire: University of Keele, 1979.

10 For a discussion of the powerful dynamics of narrative see: Newell C. The ethics of narrative ethics. Health Care Analysis 1998;6:171-4.

11 Newell C. Superhumans a super headache? Yes. The Australian 1998 March 12: 11

12 For a discussion of deaf culture see: Groce NE. Everyone here spoke sign language. Cambridge: Harvard University Press, 1985; Newell CJ. The ethics of the cochlear implant. Australian Bioethics Association 1992, Annual Conference Proceedings, vol 1. Sydney: Australian Bioethics Association, 1982; Padden C, Humphries T. Deaf in America: voices from a culture. Cambridge: Harvard University Press, 1988. Whilst I draw upon literature documenting the Australian, UK and USA deaf cultures, the material referred to can be seen to apply to each distinct culture, as the socio-linguistic analysis extends across cultures.

13 Johnson M. Aborting defective foetuses - what will it do? Link Disability fournal 1990 Aug/Sept: 14. 